\title{
ATTRACTIVE POINT THEOREMS AND ERGODIC THEOREMS FOR NONLINEAR MAPPINGS IN HILBERT SPACES
}

\author{
Lai-Jiu Lin and Wataru Takahashi
}

\begin{abstract}
In this paper, using Banach limits, we study attractive points and fixed points of nonlinear mappings in Hilbert spaces. Then we obtain attractive point theorems and fixed point theorems for nonlinear mappings in Hilbert spaces. Using these results, we finally prove a nonlinear ergodic theorem for 2-generalized hybrid mappings in Hilbert spaces.
\end{abstract}

\section{INTRODUCTION}

Throughout this paper, we denote by $\mathbb{N}$ the set of positive integers and by $\mathbb{R}$ the set of real numbers. Let $H$ be a real Hilbert space and let $C$ be a nonempty subset of $H$. Let $T$ be a mapping of $C$ into $H$. Then we denote by $F(T)$ the set of fixed points of $T$ and by $A(T)$ the set of attractive points [19] of $T$, i.e.,

(i) $F(T)=\{z \in C: T z=z\}$;

(ii) $A(T)=\{z \in H:\|T x-z\| \leq\|x-z\|, \forall x \in C\}$.

We know from [19] that $A(T)$ is closed and convex. This property is important. A mapping $T: C \rightarrow H$ is said to be nonexpansive if $\|T x-T y\| \leq\|x-y\|$ for all $x, y \in C$. We know that if $C$ is a bounded, closed and convex subset of $H$ and $T: C \rightarrow C$ is nonexpansive, then $F(T)$ is nonempty. Furthermore, from Baillon [1] we know the first nonlinear ergodic theorem in a Hilbert space. Let $C$ be a bounded,

Received March 13, 2010, accepted November 24, 2011.

Communicated by Alexander Vasiliev.

2010 Mathematics Subject Classification: 47H05, 47H09, 47H20.

Key words and phrases: Attractive point, Banach limit, Fixed point, Generalized hybrid mapping, Hilbert space, Nonexpansive mapping, 2-Generalized hybrid mapping.

The first author is partially supported by the grant of Distinguish Teaching Project of Natinal Chuanghua University of Education and NSC 99-2115-M-018-002-MY2, and the second author is also partially supported by Grant-in-Aid for Scientific Research No. 23540188 from Japan Society for the Promotion of Science. 
closed and convex subset of $H$ and let $T: C \rightarrow C$ be nonexpansive. Then for any $x \in C$,

$$
S_{n} x=\frac{1}{n} \sum_{k=0}^{n-1} T^{k} x
$$

converges weakly to an element $z \in F(T)$. An important example of nonexpansive mappings in a Hilbert space is a firmly nonexpansive mapping. A mapping $F: C \rightarrow H$ is said to be firmly nonexpansive if

$$
\|F x-F y\|^{2} \leq\langle x-y, F x-F y\rangle
$$

for all $x, y \in C$; see, for instance, Browder [2] and Goebel and Kirk [3]. Recently, Kocourek, Takahashi and Yao [7] defined a broad class of generalized hybrid mappings containing the classes of nonexpansive mappings, nonspreading mappings $[8,9]$ and hybrid mappings [18] in a Hilbert space. A mapping $T: C \rightarrow H$ is called generalized hybrid [7] if there exist $\alpha, \beta \in \mathbb{R}$ such that

$$
\alpha\|T x-T y\|^{2}+(1-\alpha)\|x-T y\|^{2} \leq \beta\|T x-y\|^{2}+(1-\beta)\|x-y\|^{2}
$$

for all $x, y \in C$. We call such a mapping an $(\alpha, \beta)$-generalized hybrid mapping. Then Kocourek, Takahashi and Yao [7] proved a fixed point theorem for such mappings in a Hilbert space. Furthermore, they proved a nonlinear mean convergence theorem of Baillon's type [1] in a Hilbert space. Maruyama, Takahashi and Yao [10] also defined a more broad class of nonlinear mappings called 2-generalized hybrid containing the class of generalized hybrid mappings. Very recently, Takahashi and Takeuchi [19] proved the following fixed point and mean convergence theorem without convexity in a Hilbert space.

Theorem 1.1. Let $H$ be a real Hilbert space and let $C$ be a nonempty subset of $H$. Let $T$ be a generalized hybrid mapping from $C$ into itself. Let $\left\{v_{n}\right\}$ and $\left\{b_{n}\right\}$ be sequences defined by

$$
v_{1} \in C, \quad v_{n+1}=T v_{n}, \quad b_{n}=\frac{1}{n} \sum_{k=1}^{n} v_{k}
$$

for all $n \in \mathbb{N}$. If $\left\{v_{n}\right\}$ is bounded, then the following hold:

(1) $A(T)$ is nonempty, closed and convex;

(2) $\left\{b_{n}\right\}$ converges weakly to $u_{0} \in A(T)$, where $u_{0}=\lim _{n \rightarrow \infty} P_{A(T)} v_{n}$ and $P_{A(T)}$ is the metric projection of $H$ onto $A(T)$.

In this paper, using Banach limits, we study attractive points and fixed points of nonlinear mappings in Hilbert spaces. Then we obtain attractive point theorems and 
fixed point theorems for nonlinear mappings in Hilbert spaces. Using these results, we finally prove a nonlinear ergodic theorem for 2-generalized hybrid mappings in Hilbert spaces.

\section{Preliminaries}

Let $H$ be a real Hilbert space with inner product $\langle\cdot \cdot\rangle$ and norm $\|\cdot\|$. We denote the strong convergence and the weak convergence of $\left\{x_{n}\right\}$ to $x \in H$ by $x_{n} \rightarrow x$ and $x_{n} \rightarrow x$, respectively. Let $A$ be a nonempty subset of $H$. We denote by $\overline{c o} A$ the closure of the convex hull of $A$. In a Hilbert space, it is known that

$$
\|\alpha x+(1-\alpha) y\|^{2}=\alpha\|x\|^{2}+(1-\alpha)\|y\|^{2}-\alpha(1-\alpha)\|x-y\|^{2}
$$

for all $x, y \in H$ and $\alpha \in \mathbb{R}$; see [17]. Furthermore, in a Hilbert space, we have that

$$
2\langle x-y, z-w\rangle=\|x-w\|^{2}+\|y-z\|^{2}-\|x-z\|^{2}-\|y-w\|^{2}
$$

for all $x, y, z, w \in H$. Indeed, we have that

$$
\begin{aligned}
2\langle x-y, z-w\rangle= & 2\langle x, z\rangle-2\langle x, w\rangle-2\langle y, z\rangle+2\langle y, w\rangle \\
= & \left(-\|x\|^{2}+2\langle x, z\rangle-\|z\|^{2}\right)+\left(\|x\|^{2}-2\langle x, w\rangle+\|w\|^{2}\right) \\
& +\left(\|y\|^{2}-2\langle y, z\rangle+\|z\|^{2}\right)+\left(-\|y\|^{2}+2\langle y, w\rangle-\|w\|^{2}\right) \\
= & \|x-w\|^{2}+\|y-z\|^{2}-\|x-z\|^{2}-\|y-w\|^{2} .
\end{aligned}
$$

From (2.2), we have that

$$
\langle(x-y)+(x-w), y-w\rangle=\|x-w\|^{2}-\|x-y\|^{2}
$$

for all $x, y, w \in H$. Let $C$ be a nonempty subset of $H$ and let $T$ be a mapping of $C$ into $H$. A mapping $T: C \rightarrow C$ is 2-generalized hybrid [10] if there exist $\alpha_{1}, \alpha_{2}, \beta_{1}, \beta_{2} \in \mathbb{R}$ such that

$$
\begin{aligned}
& \alpha_{1}\left\|T^{2} x-T y\right\|^{2}+\alpha_{2}\|T x-T y\|^{2}+\left(1-\alpha_{1}-\alpha_{2}\right)\|x-T y\|^{2} \\
& \quad \leq \beta_{1}\left\|T^{2} x-y\right\|^{2}+\beta_{2}\|T x-y\|^{2}+\left(1-\beta_{1}-\beta_{2}\right)\|x-y\|^{2}
\end{aligned}
$$

for all $x, y \in C$. We call such a mapping an $\left(\alpha_{1}, \alpha_{2}, \beta_{1}, \beta_{2}\right)$-generalized hybrid mapping. We know that the class of the mappings above covers classes of well-known mappings. For example, the class of $\left(0, \alpha_{2}, 0, \beta_{2}\right)$-generalized hybrid mappings is the class of $\left(\alpha_{2}, \beta_{2}\right)$-generalized hybrid mappings in the sense of Kocourek, Takahashi and Yao [7]. If $x=T x$ in (2.4), then for any $y \in C$,

$$
\begin{aligned}
& \alpha_{1}\|x-T y\|^{2}+\alpha_{2}\|x-T y\|^{2}+\left(1-\alpha_{1}-\alpha_{2}\right)\|x-T y\|^{2} \\
& \quad \leq \beta_{1}\|x-y\|^{2}+\beta_{2}\|x-y\|^{2}+\left(1-\beta_{1}-\beta_{2}\right)\|x-y\|^{2} .
\end{aligned}
$$


Hence we have that

$$
\|x-T y\| \leq\|x-y\|, \quad \forall x \in F(T), y \in C .
$$

This means that a 2-generalized hybrid mapping with a nonempty fixed point set is quasi-nonexpansive. The following lemma is in [15].

Lemma 2.1. Let $D$ be a nonempty, closed and convex subset of $H$ and let $f$ : $D \rightarrow(-\infty, \infty]$ be a proper, convex and lower semicontinuous function such that $f\left(z_{m}\right) \rightarrow \infty$ as $\left\|z_{m}\right\| \rightarrow \infty$. Then there exists an element $z_{0} \in D$ such that

$$
f\left(z_{0}\right)=\min \{f(z): z \in D\} .
$$

Let $l^{\infty}$ be the Banach space of bounded sequences with supremum norm. Let $\mu$ be an element of $\left(l^{\infty}\right)^{*}$ (the dual space of $\left.l^{\infty}\right)$. Then, we denote by $\mu(f)$ the value of $\mu$ at $f=\left(x_{1}, x_{2}, x_{3}, \ldots\right) \in l^{\infty}$. Sometimes, we denote by $\mu_{n}\left(x_{n}\right)$ the value $\mu(f)$. A linear functional $\mu$ on $l^{\infty}$ is called a mean if $\mu(e)=\|\mu\|=1$, where $e=(1,1,1, \ldots)$. A mean $\mu$ is called a Banach limit on $l^{\infty}$ if $\mu_{n}\left(x_{n+1}\right)=\mu_{n}\left(x_{n}\right)$. We know that there exists a Banach limit on $l^{\infty}$. If $\mu$ is a Banach limit on $l^{\infty}$, then for $f=\left(x_{1}, x_{2}, x_{3}, \ldots\right) \in l^{\infty}$,

$$
\liminf _{n \rightarrow \infty} x_{n} \leq \mu_{n}\left(x_{n}\right) \leq \limsup _{n \rightarrow \infty} x_{n} .
$$

In particular, if $f=\left(x_{1}, x_{2}, x_{3}, \ldots\right) \in l^{\infty}$ and $x_{n} \rightarrow a \in \mathbb{R}$, then we have $\mu(f)=$ $\mu_{n}\left(x_{n}\right)=a$. For the proof of existence of a Banach limit and its other elementary properties, see [15]. Using Banach limits, Takahashi and Yao [21] proved the following fixed point theorem.

Theorem 2.2. Let $H$ be a Hilbert space, let $C$ be a nonempty, closed and convex subset of $H$ and let $T$ be a mapping of $C$ into itself. Suppose that there exists an element $x \in C$ such that $\left\{T^{n} x\right\}$ is bounded and

$$
\mu_{n}\left\|T^{n} x-T y\right\|^{2} \leq \mu_{n}\left\|T^{n} x-y\right\|^{2}, \quad \forall y \in C
$$

for some Banach limit $\mu$. Then $F(T)$ is nonempty.

For proving our main results in this paper, we also need the following lemma proved by Takahashi and Toyoda [20].

Lemma 2.3. Let $D$ be a nonempty, closed and convex subset of $H$. Let $P$ be the metric projection from $H$ onto $D$. Let $\left\{u_{n}\right\}$ be a sequence in $H$. If $\left\|u_{n+1}-u\right\| \leq$ $\left\|u_{n}-u\right\|$ for any $u \in D$ and $n \in \mathbb{N}$, then $\left\{P u_{n}\right\}$ converges strongly to some $u_{0} \in D$.

The following result proved by Takahashi and Takeuchi [19] is important. 
Lemma 2.4. Let $H$ be a real Hilbert space, let $C$ be a nonempty subset of $H$ and let $T$ be a mapping from $C$ into $H$. Then $A(T)$ is a closed and convex subset of $H$.

\section{Attractive Point Theorems}

In this section, we prove an attractive point theorem for nonlinear mappings in a Hilbert space. Before proving the theorem, we show the following lemma.

Lemma 3.1. Let $H$ be a Hilbert space, let $\left\{x_{n}\right\}$ be a bounded sequence in $H$ and let $\mu$ be a mean on $l^{\infty}$. Then there exists a unique point $z_{0} \in \overline{c o}\left\{x_{n}: n \in \mathbb{N}\right\}$ such that

$$
\mu_{n}\left\langle x_{n}, y\right\rangle=\left\langle z_{0}, y\right\rangle, \quad \forall y \in H .
$$

Proof. Since $\left\{x_{n}\right\}$ is bounded, we have that for any $y \in H,\left\{\left\langle x_{n}, y\right\rangle\right\}$ is in $l^{\infty}$. Since $\mu$ is a mean on $l^{\infty}$, we can define a real valued function $g$ as follows:

$$
g(y)=\mu_{n}\left\langle x_{n}, y\right\rangle, \quad \forall y \in H .
$$

We have that for any $y, z \in H$ and $\alpha, \beta \in \mathbb{R}$,

$$
\begin{aligned}
g(\alpha y+\beta z) & =\mu_{n}\left\langle x_{n}, \alpha y+\beta z\right\rangle \\
& =\alpha \mu_{n}\left\langle x_{n}, y\right\rangle+\beta \mu_{n}\left\langle x_{n}, z\right\rangle \\
& =\alpha g(y)+\beta g(z) .
\end{aligned}
$$

Then $g$ is a linear functional of $H$ into $\mathbb{R}$. Furthermore, we have that for any $y \in H$,

$$
\begin{aligned}
|g(y)| & =\left|\mu_{n}\left\langle x_{n}, y\right\rangle\right| \\
& \leq\left\|\mu_{n}\right\| \sup _{n \in \mathbb{N}}\left|\left\langle x_{n}, y\right\rangle\right| \\
& \leq\left\|\mu_{n}\right\| \sup _{n \in \mathbb{N}}\left\|x_{n}\right\|\|y\| \\
& =\left(\sup _{n \in \mathbb{N}}\left\|x_{n}\right\|\right)\|y\| .
\end{aligned}
$$

Put $K=\sup _{n \in \mathbb{N}}\left\|x_{n}\right\|$. We have that

$$
|g(y)| \leq K\|y\|, \quad \forall y \in H .
$$

Then $g$ is bounded. By the Riesz theorem, there exists $z_{0} \in H$ such that

$$
g(y)=\left\langle z_{0}, y\right\rangle, \quad \forall y \in H .
$$

It is obvious that such $z_{0} \in H$ is unique. Furthermore, we have $z_{0} \in \overline{c o}\left\{x_{n}: n \in \mathbb{N}\right\}$. In fact, if $z_{0} \notin \overline{c o}\left\{x_{n}: n \in \mathbb{N}\right\}$, then there exists $y_{0} \in H$ from the separation theorem such that

$$
\left\langle z_{0}, y_{0}\right\rangle<\inf \left\{\left\langle z, y_{0}\right\rangle: z \in \overline{c o}\left\{x_{n}: n \in \mathbb{N}\right\}\right\} .
$$


Using the property of a mean, we have that

$$
\begin{aligned}
\left\langle z_{0}, y_{0}\right\rangle & <\inf \left\{\left\langle z, y_{0}\right\rangle: z \in \overline{c o}\left\{x_{n}: n \in \mathbb{N}\right\}\right\} \\
& \leq \inf \left\{\left\langle x_{n}, y_{0}\right\rangle: n \in \mathbb{N}\right\} \\
& \leq \mu_{n}\left\langle x_{n}, y_{0}\right\rangle \\
& =\left\langle z_{0}, y_{0}\right\rangle .
\end{aligned}
$$

This is a contradiction. Thus we have $z_{0} \in \overline{c o}\left\{x_{n}: n \in \mathbb{N}\right\}$. This completes the proof.

We call a unique $z_{0} \in H$ such that

$$
\mu_{n}\left\langle x_{n}, y\right\rangle=\left\langle z_{0}, y\right\rangle, \quad \forall y \in H
$$

the mean vector of $\left\{x_{n}\right\}$ for $\mu$.

Lemma 3.2. Let $D$ be a nonempty, closed and convex subset of a Hilbert space $H$, let $\left\{x_{n}\right\}$ be a bounded sequence in $D$ and let $\mu$ be a mean on $l^{\infty}$. If $g: D \rightarrow \mathbb{R}$ is defined by

$$
g(z)=\mu_{n}\left\|x_{n}-z\right\|^{2}, \quad \forall z \in D,
$$

then $g$ is continuous and there exists a unique $z_{0} \in D$ such that

$$
g\left(z_{0}\right)=\min \{g(z): z \in D\} .
$$

Furthermore, such $z_{0}$ is the mean vector of $\left\{x_{n}\right\}$ for $\mu$.

Proof. For a bounded sequence $\left\{x_{n}\right\} \subset D$ and a mean $\mu$ on $l^{\infty}$, we know from [15] that a function $g: D \rightarrow \mathbb{R}$ defined by

$$
g(z)=\mu_{n}\left\|x_{n}-z\right\|^{2}, \quad \forall z \in D
$$

is continuous. We also know from Lemma 3.1 that there exists the mean vector $z_{0}$ of $\left\{x_{n}\right\}$ for $\mu$, that is, there exists $z_{0} \in \overline{c o}\left\{x_{n}: n \in \mathbb{N}\right\}$ such that

$$
\mu_{n}\left\langle x_{n}, y\right\rangle=\left\langle z_{0}, y\right\rangle, \quad \forall y \in H .
$$

Since $D$ is closed and convex and $\left\{x_{n}\right\} \subset D$, we have $z_{0} \in D$. Furthermore, we have that for any $z \in D$,

$$
\begin{aligned}
g(z)-g\left(z_{0}\right) & =\mu_{n}\left\|x_{n}-z\right\|^{2}-\mu_{n}\left\|x_{n}-z_{0}\right\|^{2} \\
& =\mu_{n}\left(\left\|x_{n}-z\right\|^{2}-\left\|x_{n}-z_{0}\right\|^{2}\right) \\
& =\mu_{n}\left(\left\|x_{n}\right\|^{2}-2\left\langle x_{n}, z\right\rangle+\|z\|^{2}-\left(\left\|x_{n}\right\|^{2}-2\left\langle x_{n}, z_{0}\right\rangle+\left\|z_{0}\right\|^{2}\right)\right) \\
& =\mu_{n}\left(-2\left\langle x_{n}, z\right\rangle+\|z\|^{2}+2\left\langle x_{n}, z_{0}\right\rangle-\left\|z_{0}\right\|^{2}\right) \\
& =-2\left\langle z_{0}, z\right\rangle+\|z\|^{2}+2\left\langle z_{0}, z_{0}\right\rangle-\left\|z_{0}\right\|^{2} \\
& =-2\left\langle z_{0}, z\right\rangle+\|z\|^{2}+\left\|z_{0}\right\|^{2} \\
& =\left\|z-z_{0}\right\|^{2} .
\end{aligned}
$$


Then we have that

$$
g(z)=g\left(z_{0}\right)+\left\|z-z_{0}\right\|^{2}, \quad \forall z \in D .
$$

This implies that $z_{0}$ is a unique point in $D$ such that

$$
g\left(z_{0}\right)=\min \{g(z): z \in D\} .
$$

This completes the proof.

Now, we prove the first attractive point theorem for nonlinear mappings in a Hilbert space.

Theorem 3.3. Let $H$ be a Hilbert space, let $C$ be a nonempty subset of $H$ and let $T$ be a mapping of $C$ into itself. Suppose that there exists an element $x \in C$ such that $\left\{T^{n} x\right\}$ is bounded and

$$
\mu_{n}\left\|T^{n} x-T y\right\|^{2} \leq \mu_{n}\left\|T^{n} x-y\right\|^{2}, \quad \forall y \in C
$$

for some mean $\mu$ on $l^{\infty}$. Then $A(T)$ is nonempty. In particular, the mean vector $z_{0} \in H$ of $\left\{T^{n} x\right\}$ for $\mu$ is an element of $A(T)$. Additionally, if $C$ is closed and convex, then $F(T)$ is nonempty.

Proof. Since $\left\{T^{n} x\right\}$ is bounded, we have from Lemma 3.1 that there exists a unique point $z_{0} \in \overline{c o}\left\{T^{n} x: n \in \mathbb{N}\right\}$ such that

$$
\mu_{n}\left\langle T^{n} x, y\right\rangle=\left\langle z_{0}, y\right\rangle, \quad \forall y \in H .
$$

Using this $z_{0}$, we have from (2.3) and the assumption of $T$ that for any $v \in C$,

$$
\begin{aligned}
\left\langle\left(z_{0}-v\right)\right. & \left.+\left(z_{0}-T v\right), v-T v\right\rangle \\
& =\mu_{n}\left\langle\left(T^{n} x-v\right)+\left(T^{n} x-T v\right), v-T v\right\rangle \\
& =\mu_{n}\left(\left\|T^{n} x-T v\right\|^{2}-\left\|T^{n} x-v\right\|^{2}\right) \\
& =\mu_{n}\left\|T^{n} x-T v\right\|^{2}-\mu_{n}\left\|T^{n} x-v\right\|^{2} \\
& \leq 0 .
\end{aligned}
$$

Using (2.3) again, we have that

$$
\left\langle\left(z_{0}-v\right)+\left(z_{0}-T v\right), v-T v\right\rangle=\left\|z_{0}-T v\right\|^{2}-\left\|z_{0}-v\right\|^{2} .
$$

Thus we have that

$$
\left\|z_{0}-T v\right\|^{2}-\left\|z_{0}-v\right\|^{2} \leq 0, \quad \forall v \in C
$$

and hence

$$
\left\|z_{0}-T v\right\| \leq\left\|z_{0}-v\right\|, \quad \forall v \in C .
$$


Therefore, we have $z_{0} \in A(T)$. Additionally, if $C$ is closed and convex, we have from $\left\{T^{n} x\right\} \subset C$ that

$$
z_{0} \in \overline{c o}\left\{T^{n} x: n \in \mathbb{N}\right\} \subset C .
$$

Since $z_{0} \in A(T)$ and $z_{0} \subset C$, we have that

$$
\left\|T z_{0}-z_{0}\right\| \leq\left\|z_{0}-z_{0}\right\|=0
$$

and hence $z_{0} \in F(T)$. This completes the proof.

Using Theorem 3.3, we can prove an attractive point theorem for 2-generalized hybrid mappings in a Hilbert space.

Theorem 3.4. Let $H$ be a Hilbert space, let $C$ be a nonempty subset of $H$ and let $T$ be a 2-generalized hybrid mapping of $C$ into itself. Suppose that there exists an element $z \in C$ such that $\left\{T^{n} z\right\}$ is bounded. Then $A(T)$ is nonempty. Additionally, if $C$ is closed and convex, then $F(T)$ is nonempty.

Proof. Since a mapping $T$ is 2-generalized hybrid, there exist $\alpha_{1}, \alpha_{2}, \beta_{1}, \beta_{2} \in \mathbb{R}$ such that

$$
\begin{aligned}
& \alpha_{1}\left\|T^{2} x-T y\right\|^{2}+\alpha_{2}\|T x-T y\|^{2}+\left(1-\alpha_{1}-\alpha_{2}\right)\|x-T y\|^{2} \\
& \quad \leq \beta_{1}\left\|T^{2} x-y\right\|^{2}+\beta_{2}\|T x-y\|^{2}+\left(1-\beta_{1}-\beta_{2}\right)\|x-y\|^{2}
\end{aligned}
$$

for all $x, y \in C$. Take $z \in C$ such that $\left\{T^{n} z\right\}$ is bounded. Then for any $y \in C$ and $n \in \mathbb{N} \cup\{0\}$, we have

$$
\begin{aligned}
& \alpha_{1}\left\|T^{n+2} z-T y\right\|^{2}+\alpha_{2}\left\|T^{n+1} z-T y\right\|^{2}+\left(1-\alpha_{1}-\alpha_{2}\right)\left\|T^{n} z-T y\right\|^{2} \\
& \leq \beta_{1}\left\|T^{n+2} z-y\right\|^{2}+\beta_{2}\left\|T^{n+1} z-y\right\|^{2}+\left(1-\beta_{1}-\beta_{2}\right)\left\|T^{n} z-y\right\|^{2}
\end{aligned}
$$

for any $y \in C$. Since $\left\{T^{n} z\right\}$ is bounded, we can apply a Banach limit $\mu$ to both sides of the inequality. Then we have

$$
\begin{aligned}
& \mu_{n}\left(\alpha_{1}\left\|T^{n+2} z-T y\right\|^{2}+\alpha_{2}\left\|T^{n+1} z-T y\right\|^{2}+\left(1-\alpha_{1}-\alpha_{2}\right)\left\|T^{n} z-T y\right\|^{2}\right) \\
& \leq \mu_{n}\left(\beta_{1}\left\|T^{n+2} z-y\right\|^{2}+\beta_{2}\left\|T^{n+1} z-y\right\|^{2}+\left(1-\beta_{1}-\beta_{2}\right)\left\|T^{n} z-y\right\|^{2}\right) .
\end{aligned}
$$

So we obtain

$$
\begin{aligned}
& \alpha_{1} \mu_{n}\left\|T^{n+2} z-T y\right\|^{2}+\alpha_{2} \mu_{n}\left\|T^{n+1} z-T y\right\|^{2}+\left(1-\alpha_{1}-\alpha_{2}\right) \mu_{n}\left\|T^{n} z-T y\right\|^{2} \\
& \leq \beta_{1} \mu_{n}\left\|T^{n+2} z-y\right\|^{2}+\beta_{2} \mu_{n}\left\|T^{n+1} z-y\right\|^{2}+\left(1-\beta_{1}-\beta_{2}\right) \mu_{n}\left\|T^{n} z-y\right\|^{2}
\end{aligned}
$$

and hence

$$
\begin{aligned}
& \alpha_{1} \mu_{n}\left\|T^{n} z-T y\right\|^{2}+\alpha_{2} \mu_{n}\left\|T^{n} z-T y\right\|^{2}+\left(1-\alpha_{1}-\alpha_{2}\right) \mu_{n}\left\|T^{n} z-T y\right\|^{2} \\
& \quad \leq \beta_{1} \mu_{n}\left\|T^{n} z-y\right\|^{2}+\beta_{2} \mu_{n}\left\|T^{n} z-y\right\|^{2}+\left(1-\beta_{1}-\beta_{2}\right) \mu_{n}\left\|T^{n} z-y\right\|^{2} .
\end{aligned}
$$


This implies

$$
\mu_{n}\left\|T^{n} z-T y\right\|^{2} \leq \mu_{n}\left\|T^{n} z-y\right\|^{2}
$$

for all $y \in C$. By Theorem 2.2, we have that $A(T)$ is nonempty. Additionally, if $C$ is closed and convex, then we have from Theorem 2.2 that $F(T)$ is nonempty. This completes the proof.

As a direct consequence of Theorem 3.5, we have the attractive point theorem for generalized hybrid mappings in a Hilbert space which was proved by Takahashi and Takeuchi [19].

Theorem 3.5. Let $H$ be a Hilbert space, let $C$ be a nonempty subset of $H$ and let $T$ be a generalized hybrid mapping of $C$ into itself. Suppose that there exists an element $z \in C$ such that $\left\{T^{n} z\right\}$ is bounded. Then $A(T)$ is nonempty.

Additionally, if $C$ is closed and convex, then $F(T)$ is nonempty.

Proof. A generalized hybrid mapping $T$ of $C$ into itself is a 2-generalized hybrid mapping. That is, an $(\alpha, \beta)$-generalized hybrid mapping is a $(0, \alpha, 0, \beta)$-generalized hybrid mapping. Thus, we have the desired result from Theorem 3.5.

\section{Nonlinear ERDOdic TheOREMS}

In this section, we prove a nonlinear ergodic theorem for 2-generalized hybrid mappings without convexity in a Hilbert space. Before proving it, we obtain the following theorem.

Theorem 4.1. Let $H$ be a Hilbert space, let $C$ be a nonempty subset of $H$ and let $T$ be a mapping of $C$ into itself. Suppose that there exists an element $x \in C$ such that $\left\{T^{n} x\right\}$ is bounded and

$$
\mu_{n}\left\|T^{n} x-T y\right\|^{2} \leq \mu_{n}\left\|T^{n} x-y\right\|^{2}, \quad \forall y \in C
$$

for some Banach limit $\mu$ on $l^{\infty}$. Then $\cap_{k=1}^{\infty} \overline{c o}\left\{T^{k+n} x: n \in \mathbb{N}\right\} \cap A(T)$ consists of one point $z_{0}$. Furthermore, $z_{0}=\lim _{n \rightarrow \infty} P_{A(T)} T^{n} x$, where $P_{A(T)}$ is the metric projection of $H$ onto $A(T)$. Additionally, if $C$ is closed and convex, then $\cap_{k=1}^{\infty} \overline{c o}\left\{T^{k+n} x: n \in\right.$ $\mathbb{N}\} \cap F(T)$ consists of one point $z_{0}$.

Proof. From Lemma 3.2, a unique point $z_{0} \in H$ such that

$$
\mu_{n}\left\|T^{n} x-z_{0}\right\|^{2}=\min \left\{\mu_{n}\left\|T^{n} x-y\right\|^{2}: y \in H\right\}
$$

is the mean vector of $\left\{T^{n} x\right\}$ for the Banach limit $\mu$, that is, a unique point $z_{0} \in H$ such that

$$
\mu_{n}\left\langle T^{n} x, y\right\rangle=\left\langle z_{0}, y\right\rangle, \quad \forall y \in H .
$$


We have also from Theorem 3.3 that $z_{0} \in A(T)$. Furthermore, we have that

$$
\mu_{n}\left\|T^{n} x-z_{0}\right\|^{2}=\min \left\{\mu_{n}\left\|T^{n} x-y\right\|^{2}: y \in A(T)\right\} .
$$

Let us show that $z_{0} \in \cap_{k=1}^{\infty} \overline{c o}\left\{T^{k+n} x: n \in \mathbb{N}\right\}$. If not, there exists some $k \in \mathbb{N}$ such that $z_{0} \notin \overline{c o}\left\{T^{k+n} x: n \in \mathbb{N}\right\}$. By the separation theorem, there exists $y_{0} \in H$ such that

$$
\left\langle z_{0}, y_{0}\right\rangle<\inf \left\{\left\langle z, y_{0}\right\rangle: z \in \overline{c o}\left\{T^{k+n} x: n \in \mathbb{N}\right\}\right\} .
$$

Using the property of a Banach limit, we have that

$$
\begin{aligned}
\left\langle z_{0}, y_{0}\right\rangle & <\inf \left\{\left\langle z, y_{0}\right\rangle: z \in \overline{c o}\left\{T^{k+n} x: n \in \mathbb{N}\right\}\right\} \\
& \leq \inf \left\{\left\langle T^{k+n} x, y_{0}\right\rangle: n \in \mathbb{N}\right\} \\
& \leq \mu_{n}\left\langle T^{k+n} x, y_{0}\right\rangle \\
& =\mu_{n}\left\langle T^{n} x, y_{0}\right\rangle \\
& =\left\langle z_{0}, y_{0}\right\rangle .
\end{aligned}
$$

This is a contradiction. Thus, we have that $z_{0} \in \cap_{k=1}^{\infty} \overline{c o}\left\{T^{k+n} x: n \in \mathbb{N}\right\}$. Next, we show that $\cap_{k=1}^{\infty} \overline{c o}\left\{T^{k+n} x: n \in \mathbb{N}\right\} \cap A(T)$ consists of one point $z_{0}$. Assume that $z_{1} \in \cap_{k=1}^{\infty} \overline{c o}\left\{T^{k+n} x: n \in \mathbb{N}\right\} \cap A(T)$. Since $z_{1} \in A(T)$, we have that

$$
\left\|T^{n+1} x-z_{1}\right\|^{2} \leq\left\|T^{n} x-z_{1}\right\|^{2}, \quad \forall n \in \mathbb{N} .
$$

Then $\lim _{n \rightarrow \infty}\left\|T^{n} x-z_{1}\right\|^{2}$ exists. In general, since $\lim _{n \rightarrow \infty}\left\|T^{n} x-z\right\|^{2}$ exists for every $z \in A(T)$, we define a function $g: A(T) \rightarrow \mathbb{R}$ as follows:

$$
g(z)=\lim _{n \rightarrow \infty}\left\|T^{n} x-z\right\|^{2}, \quad \forall z \in A(T) .
$$

Since

$$
\left\|z_{0}-z_{1}\right\|^{2}=\left\|T^{n} x-z_{1}\right\|^{2}-\left\|T^{n} x-z_{0}\right\|^{2}-2\left\langle z_{0}-z_{1}, T^{n} x-z_{0}\right\rangle
$$

for every $n \in \mathbb{N}$, we have

$$
\begin{aligned}
\left\|z_{0}-z_{1}\right\|^{2}+2 & \lim _{n \rightarrow \infty}\left\langle z_{0}-z_{1}, T^{n} x-z_{0}\right\rangle \\
& =\lim _{n \rightarrow \infty}\left\|T^{n} x-z_{1}\right\|^{2}-\lim _{n \rightarrow \infty}\left\|T^{n} x-z_{0}\right\|^{2} \\
& \geq 0 .
\end{aligned}
$$

Let $\epsilon>0$. Then we have

$$
2 \lim _{n \rightarrow \infty}\left\langle z_{0}-z_{1}, T^{n} x-z_{0}\right\rangle>-\left\|z_{0}-z_{1}\right\|^{2}-\epsilon .
$$


Hence, there exists $n_{0} \in \mathbb{N}$ such that

$$
2\left\langle z_{0}-z_{1}, T^{n} x-z_{0}\right\rangle>-\left\|z_{0}-z_{1}\right\|^{2}-\epsilon
$$

for every $n \in \mathbb{N}$ with $n \geq n_{0}$. Since $z_{1} \in \cap_{k=1}^{\infty} \overline{c o}\left\{T^{k+n} x: n \in \mathbb{N}\right\}$, we have

$$
2\left\langle z_{0}-z_{1}, z_{1}-z_{0}\right\rangle \geq-\left\|z_{0}-z_{1}\right\|^{2}-\epsilon .
$$

This inequality implies that $\left\|z_{0}-z_{1}\right\|^{2} \leq \epsilon$. Since $\epsilon>0$ is arbitrary, we have $z_{0}=z_{1}$. Therefore,

$$
\left\{z_{0}\right\}=\cap_{k=1}^{\infty} \overline{c o}\left\{T^{k+n} x: n \in \mathbb{N}\right\} \cap A(T) .
$$

We show that $z_{0}=\lim _{n \rightarrow \infty} P_{A(T)} T^{n} x$, where $P_{A(T)}$ is the metric projection of $H$ onto $A(T)$. Since

$$
\left\|T^{n+1} x-z\right\| \leq\left\|T^{n} x-z\right\|, \quad \forall n \in \mathbb{N}, z \in A(T),
$$

we have from Lemma 2.3 that $\left\{P_{A(T)} T^{n} x\right\}$ converges strongly to some $u \in A(T)$. Since $P_{A(T)} T^{n} x \in A(T)$ for all $n \in \mathbb{N}$, we have

$$
\left\|P_{A(T)} T^{m} x-T^{n} x\right\| \leq\left\|P_{A(T)} T^{m} x-T^{m} x\right\|
$$

for all $n, m \in \mathbb{N}$ with $n \geq m$. Furthermore, we have from the property of $P_{A(T)}$ that

$$
\left\|P_{A(T)} T^{m} x-T^{m} x\right\| \leq\left\|z-T^{m} x\right\|
$$

for all $z \in A(T)$. Thus,

$$
\left\|P_{A(T)} T^{m} x-T^{n} x\right\|^{2} \leq\left\|P_{A(T)} T^{m} x-T^{m} x\right\|^{2} \leq\left\|z-T^{m} x\right\|^{2}
$$

for all $n, m \in \mathbb{N}$ with $n \geq m$ and $z \in A(T)$. Then we have that

$$
\begin{aligned}
g\left(P_{A(T)} T^{m} x\right) & =\lim _{n \rightarrow \infty}\left\|P_{A(T)} T^{m} x-T^{n} x\right\|^{2} \\
& \leq\left\|P_{A(T)} T^{m} x-T^{m} x\right\|^{2} \\
& \leq\left\|z-T^{m} x\right\|^{2} .
\end{aligned}
$$

Since $g$ is continuous and $P_{A(T)} T^{m} x \rightarrow u \in A(T)$, we have that

$$
g(u) \leq \lim _{m \rightarrow \infty}\left\|z-T^{m} x\right\|^{2}=g(z), \quad \forall z \in A(T) .
$$

Since $z_{0}$ is a unique minimizer of $g$ in $A(T)$, we have $u=z_{0}$. Therefore,

$$
z_{0}=\lim _{n \rightarrow \infty} P_{A(T)} T^{n} x .
$$


Additionally, if $C$ is closed and convex, then we know that

$$
z_{0} \in \cap_{k=1}^{\infty} \overline{c o}\left\{T^{k+n} x: n \in \mathbb{N}\right\} \cap F(T) .
$$

Since $\cap_{k=1}^{\infty} \overline{c o}\left\{T^{k+n} x: n \in \mathbb{N}\right\} \cap A(T)$ consists of one point $z_{0}$, we have

$$
\cap_{k=1}^{\infty} \overline{c o}\left\{T^{k+n} x: n \in \mathbb{N}\right\} \cap F(T)=\left\{z_{0}\right\} .
$$

This completes the proof.

Lemma 4.2. Let $H$ be a Hilbert space, let $C$ be a nonempty subset of $H$ and let $T$ be a mapping of $C$ into itself. Suppose that there exists an element $x \in C$ such that $\left\{T^{n} x\right\}$ is bounded and

$$
\mu_{n}\left\|T^{n} x-T y\right\|^{2} \leq \mu_{n}\left\|T^{n} x-y\right\|^{2}, \quad \forall y \in C
$$

for some Banach limit $\mu$ on $l^{\infty}$. Define

$$
S_{n} x=\frac{1}{n} \sum_{k=0}^{n-1} T^{k} x, \quad \forall n \in \mathbb{N} .
$$

Suppose that if a subsequence $\left\{S_{n_{i}} x\right\}$ of $\left\{S_{n} x\right\}$ converges weakly to $v \in H$, then $v \in$ $A(T)$. Then $\left\{S_{n} x\right\}$ converges weakly to $z_{0} \in A(T)$, where $z_{0}=\lim _{n \rightarrow \infty} P_{A(T)} T^{n} x$. Additionally, if $C$ is closed and convex, then $\left\{S_{n} x\right\}$ converges weakly to $z_{0} \in F(T)$.

Proof. From Theorem 4.1, we know that $\cap_{k=1}^{\infty} \overline{c o}\left\{T^{k+n} x: n \in \mathbb{N}\right\} \cap A(T)$ consists of one point $z_{0}$ and $z_{0}=\lim _{n \rightarrow \infty} P_{A(T)} T^{n} x$. To complete the proof, it is sufficient to show that if $S_{n_{i}} x \rightarrow v$, then $v=z_{0}$. Since $P_{A(T)} T^{n} x \in A(T)$ for all $n \in \mathbb{N}$, we have

$$
\begin{aligned}
\left\|P_{A(T)} T^{n} x-T^{n} x\right\| & \leq\left\|P_{A(T)} T^{n-1} x-T^{n} x\right\| \\
& \leq\left\|P_{A(T)} T^{n-1} x-T^{n-1} x\right\| .
\end{aligned}
$$

This implies that $\left\{\left\|P_{A(T)} T^{n} x-T^{n} x\right\|\right\}$ is nonincreasing. We have also that

$$
\left\langle T^{k} x-P_{A(T)} T^{k} x, P_{A(T)} T^{k} x-u\right\rangle \geq 0
$$

for all $k \in \mathbb{N}$ and $u \in A(T)$. Since $\left\{\left\|T^{k} x-P_{A(T)} T^{k} x\right\|\right\}$ is nonincreasing, we have

$$
\begin{aligned}
\left\langle u-z_{0}, T^{k} x-P_{A(T)} T^{k} x\right\rangle & \leq\left\langle P_{A(T)} T^{k} x-z_{0}, T^{k} x-P_{A(T)} T^{k} x\right\rangle \\
& \leq\left\|P_{A(T)} T^{k} x-z_{0}\right\| \cdot\left\|T^{k} x-P_{A(T)} T^{k} x\right\| \\
& \leq\left\|P_{A(T)} T^{k} x-z_{0}\right\| \cdot\left\|x-P_{A(T)} x\right\| .
\end{aligned}
$$


Adding these inequalities from $k=0$ to $k=n-1$ and dividing $n$, we have

$$
\left\langle u-z_{0}, S_{n} x-\frac{1}{n} \sum_{k=0}^{n-1} P_{A(T)} T^{k} x\right\rangle \leq \frac{\left\|x-P_{A(T)} x\right\|}{n} \sum_{k=0}^{n-1}\left\|P_{A(T)} T^{k} x-z_{0}\right\| .
$$

Since $S_{n_{i}} x \rightarrow v$ and $P_{A(T)} T^{k} x \rightarrow z_{0}$, we have

$$
\left\langle u-z_{0}, v-z_{0}\right\rangle \leq 0
$$

By the assumption, we know $v \in A(T)$. Putting $u=v$, we have $\left\langle v-z_{0}, v-z_{0}\right\rangle \leq 0$ and hence $\left\|v-z_{0}\right\|^{2} \leq 0$. Thus, we obtain $v=z_{0}$. Therefore, $\left\{S_{n} x\right\}$ converges weakly to $z_{0} \in A(T)$. Additionally, if $C$ is closed and convex, then we know from Theorem 4.1 that $z_{0}$ is a fixed point. This completes the proof.

Now, we can prove the following nonlinear ergodic theorem for 2-generalized hybrid mappings in a Hilbert space.

Theorem 4.3. Let $H$ be a Hilbert space, let $C$ be a nonempty subset of $H$ and let $T$ be a 2-generalized hybrid mapping of $C$ into itself such that $A(T)$ is nonempty. Then for any $x \in C$,

$$
S_{n} x=\frac{1}{n} \sum_{k=0}^{n-1} T^{k} x
$$

converges weakly to $z_{0} \in A(T)$, where $z_{0}=\lim _{n \rightarrow \infty} P_{A(T)} T^{n} x$. Additionally, if $C$ is closed and convex, then $\left\{S_{n} x\right\}$ converges weakly to $z_{0} \in F(T)$, where $z_{0}=$ $\lim _{n \rightarrow \infty} P_{F(T)} T^{n} x$.

Proof. Since $T: C \rightarrow C$ is 2-generalized hybrid, there exist $\alpha_{1}, \alpha_{2}, \beta_{1}, \beta_{2} \in \mathbb{R}$ such that

$$
\begin{aligned}
\alpha_{1} \| T^{2} x & -T y\left\|^{2}+\alpha_{2}\right\| T x-T y\left\|^{2}+\left(1-\alpha_{1}-\alpha_{2}\right)\right\| x-T y \|^{2} \\
& \leq \beta_{1}\left\|T^{2} x-y\right\|^{2}+\beta_{2}\|T x-y\|^{2}+\left(1-\beta_{1}-\beta_{2}\right)\|x-y\|^{2}
\end{aligned}
$$

for all $x, y \in C$. Since $A(T)$ is nonempty, $\left\{T^{n} x\right\}$ is bounded for any $x \in C$. We know from the proof of Theorem 3.5 that for any Banach limits $\mu$ on $l^{\infty}$,

$$
\mu_{n}\left\|T^{n} x-T y\right\|^{2} \leq \mu_{n}\left\|T^{n} x-y\right\|^{2}, \quad \forall y \in C .
$$

Thus we have from Theorem 4.1 that $\cap_{k=1}^{\infty} \overline{c o}\left\{T^{k+n} x: n \in \mathbb{N}\right\} \cap A(T)$ consists of one point $z_{0}$ and $z_{0}=\lim _{n \rightarrow \infty} P_{A(T)} T^{n} x$, where $P_{A(T)}$ is the metric projection of $H$ onto $A(T)$. To prove $S_{n} x \rightarrow z_{0} \in A(T)$, it is sufficient to show from Lemma 4.2 that 
if $S_{n_{i}} x \rightarrow v$, then $v \in A(T)$. Assume $S_{n_{i}} x \rightarrow v$. We have from (4.1) that for any $y \in C$ and $k \in \mathbb{N} \cup\{0\}$

$$
\begin{aligned}
0 \leq & \beta_{1}\left\|T^{k+2} x-y\right\|^{2}+\beta_{2}\left\|T^{k+1} x-y\right\|^{2}+\left(1-\beta_{1}-\beta_{2}\right)\left\|T^{k} x-y\right\|^{2} \\
& -\alpha_{1}\left\|T^{k+2} x-T y\right\|^{2}-\alpha_{2}\left\|T^{k+1} x-T y\right\|^{2}-\left(1-\alpha_{1}-\alpha_{2}\right)\left\|T^{k} x-T y\right\|^{2} \\
= & \beta_{1}\left(\left\|T^{k+2} x-T y\right\|^{2}+2\left\langle T^{k+2} x-T y, T y-y\right\rangle+\|T y-y\|^{2}\right) \\
& +\beta_{2}\left(\left\|T^{k+1} x-T y\right\|^{2}+2\left\langle T^{k+1} x-T y, T y-y\right\rangle+\|T y-y\|^{2}\right) \\
& +\left(1-\beta_{1}-\beta_{2}\right)\left(\left\|T^{k} x-T y\right\|^{2}+2\left\langle T^{k} x-T y, T y-y\right\rangle+\|T y-y\|^{2}\right) \\
& -\alpha_{1}\left\|T^{k+2} x-T y\right\|^{2}-\alpha_{2}\left\|T^{k+1} x-T y\right\|^{2}-\left(1-\alpha_{1}-\alpha_{2}\right)\left\|T^{k} x-T y\right\|^{2} \\
= & \|T y-y\|^{2}+2\left\langle\beta_{1} T^{k+2} x+\beta_{2} T^{k+1} x+\left(1-\beta_{1}-\beta_{2}\right) T^{k} x-T y, T y-y\right\rangle \\
& +\left(\beta_{1}-\alpha_{1}\right)\left(\left\|T^{k+2} x-T y\right\|^{2}-\left\|T^{k} x-T y\right\|^{2}\right) \\
& +\left(\beta_{2}-\alpha_{2}\right)\left(\left\|T^{k+1} x-T y\right\|^{2}-\left\|T^{k} x-T y\right\|^{2}\right) .
\end{aligned}
$$

Summing up these inequalities with respect to $k=0,1, \ldots, n-1$,

$$
\begin{aligned}
0 \leq & n\|T y-y\|^{2} \\
& +2\left\langle\sum_{k=0}^{n-1} T^{k} x+\beta_{1}\left(T^{n+1} x+T^{n} x-x-T x\right)+\beta_{2}\left(T^{n} x-x\right)-n T y, T y-y\right\rangle \\
& +\left(\beta_{1}-\alpha_{1}\right)\left(\left\|T^{n+1} x-T y\right\|^{2}+\left\|T^{n} x-T y\right\|^{2}-\|x-T y\|^{2}-\|T x-T y\|^{2}\right) \\
& +\left(\beta_{2}-\alpha_{2}\right)\left(\left\|T^{n} x-T y\right\|^{2}-\|x-T y\|^{2}\right) .
\end{aligned}
$$

Deviding this inequality by $n$, we have

$$
\begin{aligned}
0 \leq & \|T y-y\|^{2} \\
& +2\left\langle S_{n} x+\frac{1}{n} \beta_{1}\left(T^{n+1} x+T^{n} x-x-T x\right)+\frac{1}{n} \beta_{2}\left(T^{n} x-x\right)-T y, T y-y\right\rangle \\
& +\frac{1}{n}\left(\beta_{1}-\alpha_{1}\right)\left(\left\|T^{n+1} x-T y\right\|^{2}+\left\|T^{n} x-T y\right\|^{2}-\|x-T y\|^{2}-\|T x-T y\|^{2}\right) \\
& +\frac{1}{n}\left(\beta_{2}-\alpha_{2}\right)\left(\left\|T^{n} x-T y\right\|^{2}-\|x-T y\|^{2}\right) .
\end{aligned}
$$

Replacing $n$ by $n_{i}$ and letting $n_{i} \rightarrow \infty$, we obtain from $S_{n_{i}} x \rightarrow v$ that

$$
0 \leq\|T y-y\|^{2}+2\langle v-T y, T y-y\rangle .
$$

Using (2.2), we have that

$$
\begin{aligned}
0 & \leq\|T y-y\|^{2}+2\langle v-T y, T y-y\rangle \\
& =\|T y-y\|^{2}+\|v-y\|^{2}-\|v-T y\|^{2}-\|T y-y\|^{2} \\
& =\|v-y\|^{2}-\|v-T y\|^{2} .
\end{aligned}
$$


Thus we have that

$$
\|v-T y\| \leq\|v-y\|, \quad \forall y \in C
$$

and hence $v \in A(T)$. Therefore $S_{n} x \rightarrow z_{0} \in A(T)$. Assume that $C$ is closed and convex. Since $T$ is 2-generalized hybrid, $T$ is quasi-nonexpansive from (2.5). Then we have that $F(T)$ is closed and convex; see Ito and Takahashi [6]. Thus we can define the metric projection $P_{F(T)}$ of $H$ onto $F(T)$. Since $C$ is closed and convex, $\left\{S_{n} x\right\}$ converges weakly to $z_{0} \in F(T)$. To show $z_{0}=\lim _{n \rightarrow \infty} P_{F(T)} T^{n} x$, we may follow the proof of Theorem 4.1. This completes the proof.

As a direct consequence of Theorem 4.3, we have the nonlinear ergodic theorem by Takahashi and Takeuchi [19].

Theorem 4.4. Let $H$ be a Hilbert space, let $C$ be a nonempty subset of $H$ and let $T$ be a generalized hybrid mapping of $C$ into itself such that $A(T)$ is nonempty. Then for any $x \in C$,

$$
S_{n} x=\frac{1}{n} \sum_{k=0}^{n-1} T^{k} x
$$

converges weakly to $z_{0} \in A(T)$, where $z_{0}=\lim _{n \rightarrow \infty} P_{A(T)} T^{n} x$. Additionally, if $C$ is closed and convex, then $\left\{S_{n} x\right\}$ converges weakly to $z_{0} \in F(T)$, where $z_{0}=$ $\lim _{n \rightarrow \infty} P_{F(T)} T^{n} x$.

\section{REFERENCES}

1. J.-B. Baillon, Un theoreme de type ergodique pour les contractions non lineaires dans un espace de Hilbert, C. R. Acad. Sci. Paris Ser. A-B, 280 (1975), 1511-1514.

2. F. E. Browder, Convergence theorems for sequences of nonlinear operators in Banach spaces, Math. Z., 100 (1967), 201-225.

3. K. Goebel and W. A. Kirk, Topics in Metric Fixed Point Theory, Cambridge University Press, Cambridge, 1990.

4. K. Goebel and S. Reich, Uniform Convexity, Hyperbolic Geometry, and Nonexpansive Mappings, Marcel Dekker Inc., New York, 1984.

5. S. Iemoto and $\mathrm{W}$. Takahashi, Approximating fixed points of nonexpansive mappings and nonspreading mappings in a Hilbert space, Nonlinear Anal., 71 (2009), 2082-2089.

6. S. Ito and W. Takahashi, The common fixed points theory of single-valued mappings and multi-valued mappings, Pacific J. Math., 79 (1978), 493-508.

7. P. Kocourek, W. Takahashi and J.-C. Yao, Fixed point theorems and weak convergence theorems for genelalized hybrid mappings in Hilbert spaces, Taiwanese J. Math., 14 (2010), 2497-2511. 
8. F. Kohsaka and W. Takahashi, Existence and approximation of fixed points of firmly nonexpansive-type mappings in Banach spaces, SIAM J. Optim., 19 (2008), 824-835.

9. F. Kohsaka and W. Takahashi, Fixed point theorems for a class of nonlinear mappings related to maximal monotone operators in Banach spaces, Arch. Math., 91 (2008), 166-177.

10. T. Maruyama, W. Takahashi and M. Yao, Fixed point and mean ergodic theorems for new nonlinear mappings in Hilbert spaces, J. Nonlinear Convex Anal., 12 (2011), 185-197.

11. Z. Opial, Weak convergence of the sequence of successive approximations for nonexpansive mappings, Bull. Amer. Math. Soc., 73 (1967), 591-597.

12. S. Reich and D. Shoikhet, Nonlinear Semigroups, Fixed points, and Geometry of Domains in Banach Spaces, Imperial College Press, London, 2005.

13. W. Takahashi, A nonlinear ergodic theorem for an amenable semigroup of nonexpansive mappings in a Hilbert space, Proc. Amer. Math. Soc., 81 (1981), 253-256.

14. W. Takahashi, A nonlinear ergodic theorem for a reversible semigroup of nonexpansive mappings in a Hilbert space, Proc. Amer. Math. Soc., 97 (1986), 55-58.

15. W. Takahashi, Nonlinear Functional Analysis. Fixed Point Theory and its Applications, Yokohama Publishers, Yokohama, 2000.

16. W. Takahashi, Convex Analysis and Approximation of Fixed Points (Japanese), Yokohama Publishers, Yokohama, 2000.

17. W. Takahashi, Introduction to Nonlinear and Convex Analysis, Yokohama Publishers, Yokohama, 2009.

18. W. Takahashi, Fixed point theorems for new nonlinear mappings in a Hilbert space, $J$. Nonlinear Convex Anal., 11 (2010), 79-88.

19. W. Takahashi and Y. Takeuchi, Nonlinear ergodic theorem without convexity for generalized hybrid mappings in a Hilbert space, J. Nonlinear Convex Anal., 12 (2011), 399-406.

20. W. Takahashi and M. Toyoda, Weak convergence theorems for nonexpansive mappings and monotone mappings, J. Optim. Theory Appl., 118 (2003), 417-428.

21. W. Takahashi and J.-C. Yao, Fixed point theorems and ergodic theorems for nonlinear mappings in Hilbert spaces, Taiwanese J. Math., 15 (2011), 457-472.

Lai-Jiu Lin

Department of Mathematics

National Changhua University of Education

Changhua, Taiwan

E-mail: maljlin@cc.ncu.edu.tw 


\author{
Wataru Takahashi \\ Department of Mathematical and Computing Sciences \\ Tokyo Institute of Technology \\ Tokyo 152-8552, Japan \\ and \\ Department of Mathematics \\ National Changhua University of Education \\ Changhua, Taiwan \\ E-mail: wataru@is.titech.ac.jp
}


